\title{
Two Photon Induced Lasing in 1550 nm Quantum Dash Optical Gain Media
}

\author{
Capua, Amir; Saal, Abigael; Reithmaier, Johann Peter; Yvind, Kresten; Eisenstein, Gad
}

Published in:

Proceedings of the European Conference on Optical Communication (ECOC) 2011

Publication date:

2011

Document Version

Publisher's PDF, also known as Version of record

Link back to DTU Orbit

Citation (APA):

Capua, A., Saal, A., Reithmaier, J. P., Yvind, K., \& Eisenstein, G. (2011). Two Photon Induced Lasing in 1550 $\mathrm{nm}$ Quantum Dash Optical Gain Media. In Proceedings of the European Conference on Optical Communication (ECOC) 2011 (pp. Tu.6.LeSaleve). Optical Society of America. http://www.ecoc2011.org/

\section{General rights}

Copyright and moral rights for the publications made accessible in the public portal are retained by the authors and/or other copyright owners and it is a condition of accessing publications that users recognise and abide by the legal requirements associated with these rights.

- Users may download and print one copy of any publication from the public portal for the purpose of private study or research.

- You may not further distribute the material or use it for any profit-making activity or commercial gain

- You may freely distribute the URL identifying the publication in the public portal 


\title{
Two Photon Induced Lasing in 1550 nm Quantum Dash Optical Gain Media
}

\author{
A. Capua ${ }^{1}$, A. Saal ${ }^{1}$, J.P. Reithmaier ${ }^{2}$, K. Yvind ${ }^{3}$, G. Eisenstein ${ }^{1}$ \\ ${ }^{I}$ Electrical Engineering Department, Technion, Haifa 32000 Israel \\ ${ }^{2}$ Technische Physik, Institute of Nanostructure Technologies and Analytics, University Kassel, Germany \\ ${ }^{3}$ Department of Photonics Engineering, DTU-Fotonik, Technical University of Denmark,2800 Kgs. Lyngby, Denmark \\ Author e-mail address: acapua@tx.technion.ac.il
}

\begin{abstract}
We report on a unique lasing mechanism observed in quantum dash Gain media. While the gain media is electrically pumped below lasing threshold, a strong optical pulse excites carriers by two photon absorption into high energy states of the quantum dashes and wetting layer. Fast inter band carrier relaxation and capture processes into the ground states of the quantum dashes result in increased gain followed by lasing at the gain peak irrespective of the stimulating pulse wavelength. The temporal response of the lasing line is examined on a $40 \mathrm{GHz}$ scope and full characterization of the pulse by the XFROG scheme is performed. We show the lasing mechanism to be governed mainly by the wetting layer dynamics and extract a direct measurement of the carrier-carrier scattering time constant.

OCIS codes: (140.5960) Semiconductor lasers; (250.5980) Semiconductor optical amplifiers; (230.5590) Quantumwell, -wire and -dot devices
\end{abstract}

A unique phenomenon in which an instantaneous gain response takes place in quantum dash (QDash) optical amplifiers was recently reported [1]. This process is initiated by high energy carriers, induced by two photon absorption (TPA), which relax fast to the ground states of all QDashes via carrier-carrier scattering. The instantaneous gain occurs all across the inhomogeneously broadened gain spectrum and is observable using multi wavelength pump probe measurements.

This paper describes a unique use of the TPA induced gain which can be sufficiently large to cause a QDash amplifier with low, but finite facet reflectivities, to oscillate with a threshold that depends on the optical pulse wavelength. The laser oscillation was characterized in both the spectral and time domains. The gain inducing pulse itself was characterized using X-FROG measurements which yield pulse shape and phase responses on the time scale of the, $150 \mathrm{fs}$ wide, pulse itself.

The QDash amplifier we used was $1.5 \mathrm{~mm}$ long. The gain region comprised six InAs QDash layers separated by InGaAlAs barriers placed within a GRINCH structure. The amplifier facet reflectivities were estimated to be $0.03 \%$ $-0.05 \%$.
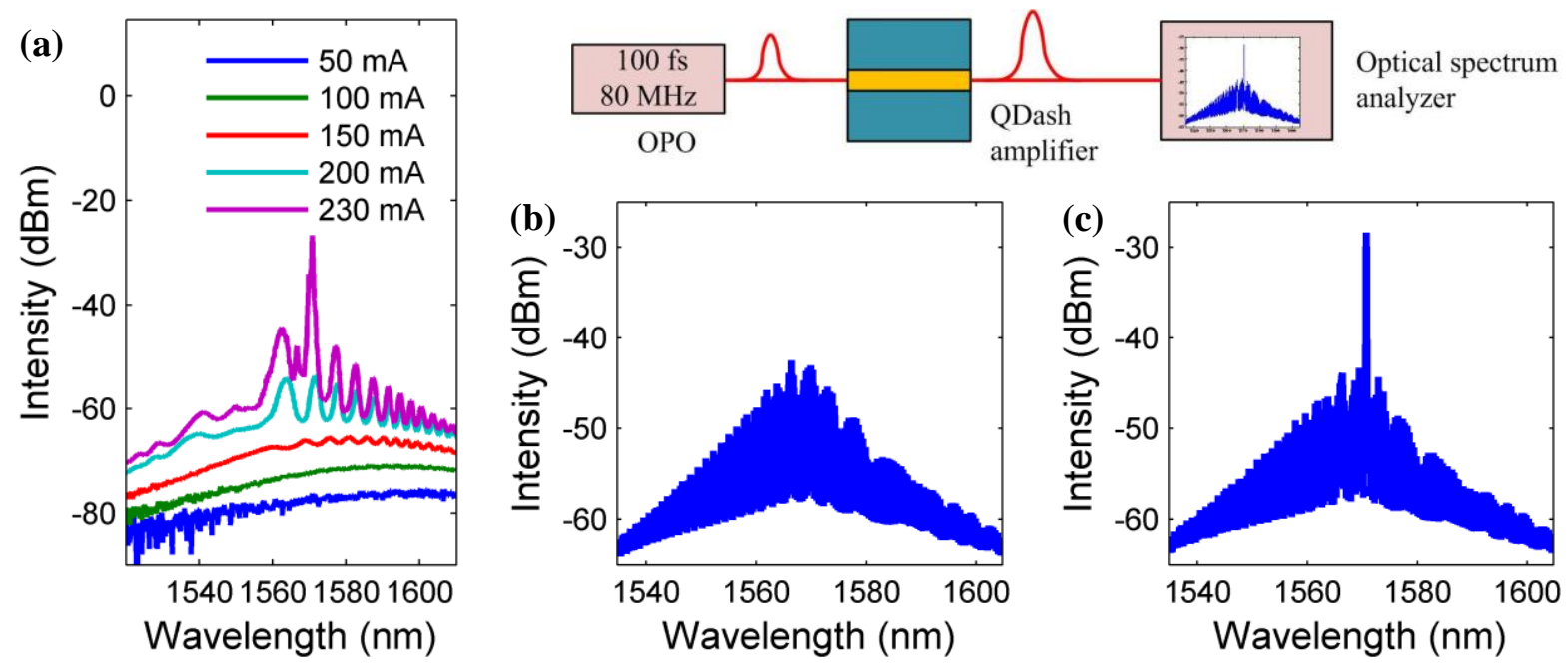

Fig. 1 - Measured spectra. (a) Bias dependent ASE. (b) ASE at a bias of $210 \mathrm{~mA}$. (c) Output spectrum at a bias of $210 \mathrm{~mA}$ fed by a $100 \mathrm{fs}$, $300 \mathrm{pJ}$ optical pump at $1530 \mathrm{~nm}$ as shown above the spectra. 
The bias dependent amplified spontaneous emission spectra are shown in Fig. 1(a). For bias currents above $220 \mathrm{~mA}$ (which is 5.5 times its lasing threshold prior to the facet coating), the amplifier lases. Driving the amplifier at 210 $\mathrm{mA}$ yields the ASE spectrum shown in Fig. 1(b). Adding an optical input pulse (as seen in the schematic above Fig. $3(\mathrm{c})$ ) yields a clear lasing spectrum with a narrow line at $1570 \mathrm{~nm}$ which is $15 \mathrm{~dB}$ larger than the background. The lasing line appears always at the same spectral location independent of the pump wavelength. In order to understand the lasing phenomenon we examined the bias threshold for different pump wavelengths keeping the pump energy constant at 3 pJ. The results are shown in Fig. 2 (a).
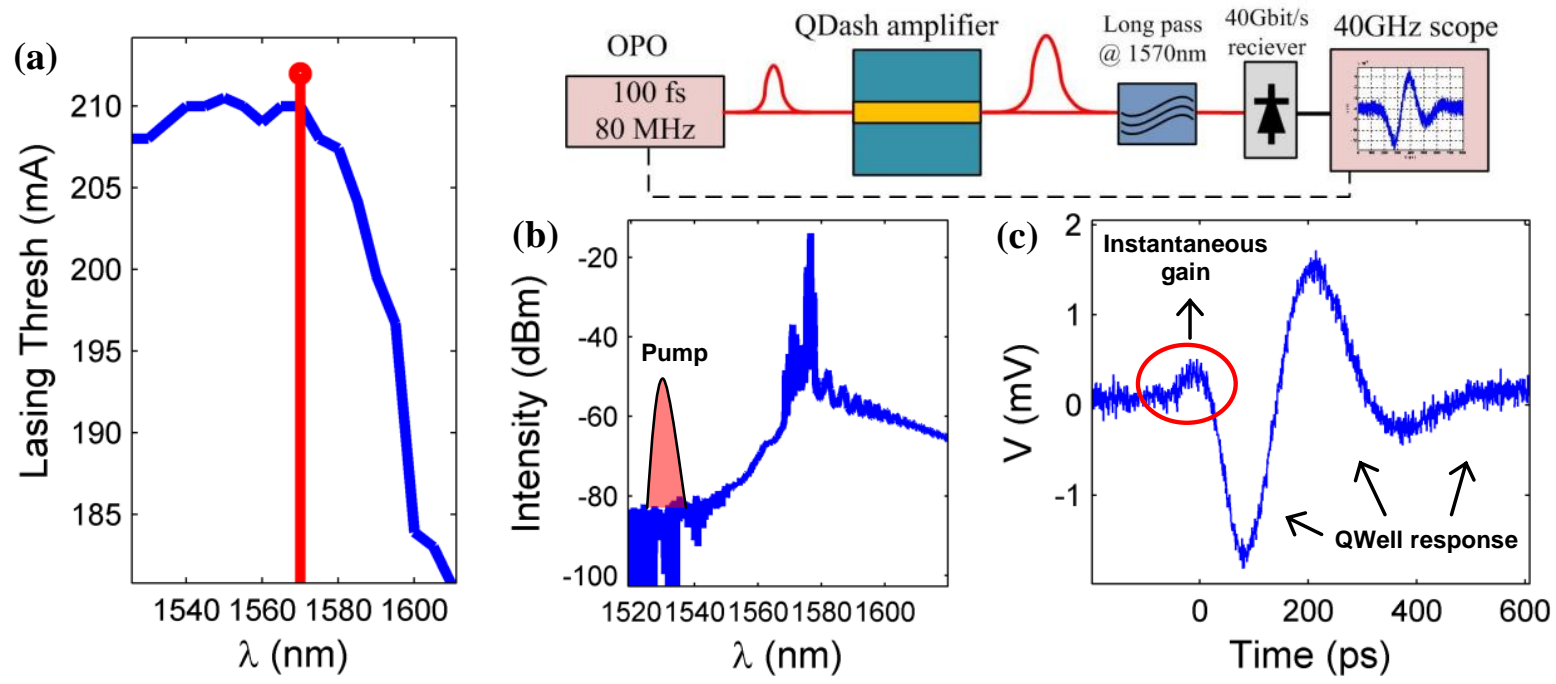

Fig. 2 - (a) Wavelength dependent threshold for a fixed pump power of 3 pJ. (b) Resolved spectrum after long pass filter. The filtered pump is marked for illustration. (c) Temporal response of the laser line. Time response measurement setup is shown above.

The lasing wavelength, $1570 \mathrm{~nm}$ is marked by a red line. For pump wavelengths shorter than $1570 \mathrm{~nm}$, the threshold is basically constant at $210 \mathrm{~mA}$. However, for long pump wavelengths, the lasing threshold drops significantly down to $180 \mathrm{~mA}$ at $1608 \mathrm{~nm}$.

The optically induced lasing results from a TPA induced gain increase as demonstrated in [1]. This gain increase has an instantaneous response which is irrelevant to the lasing phenomenon but has also a long lived component due to an increase of the barrier state and wetting layer populations. This long lived component manifests itself in the pump probe measurements [1] as an increased transmission, relative to the transmission prior to the pulse perturbation, which lasts for many tens of ps or more. This is long enough for several round trips in the $1.5 \mathrm{~mm}$ long amplifier so that lasing oscillation can build up. This means of course that the spectrum in Fig. 1(c) is an average spectrum and the laser is on for only a fraction of the time between the pump pulses (12.5 ns). With this concept in mind, it is possible to explain Fig. 2(a). The longer the pump wavelength, the lower the energy of the TPA induced carriers and therefore the energetic distance to the lasing wavelength $(1570 \mathrm{~nm}$, where the cavity losses are minimal) is smaller. This means that the carrier relaxation to the lasing state, mediated by carrier-carrier scattering, is faster $[2,3]$ so the gain increase is more efficient. Moreover, pumping at long wavelengths causes no depletion of carriers at energies higher than the lasing energy thereby maintaining the efficiency of the carrier-carrier scattering relaxation. The results of these two effects is a reduction of threshold with pump wavelength as seen in Fig. 2(a).

To clarify the effect further, we performed spectrally resolved time response measurements. An example is shown in Fig. 2(b) and 2(c). Fig. 2(b) shows the spectral constellation of the pump (this time at $1530 \mathrm{~nm}$ ) and the lasing line which is $40 \mathrm{~nm}$ longer. The lasing line was filtered using the set up shown above Fig. 2(c) and, detected using a fast detector and measured on a fast sampling oscilloscope. The result is shown in Fig. 2(c) which shows an initial (unresolved) instantaneous gain increase circled in red followed by rather conventional relaxation oscillations of the laser as it turns on.

Several experiments measuring the temporal response of semiconductor lasers biased above lasing threshold and 
perturbed by an optical pulse were previously performed [4-7]. These were performed in order to study relaxation oscillations and laser line gain switching and yielded a variety of interesting phenomena such as large signal optical modulation [6] and the initiation of dark pulses [7]. however, no previous report showed any evidence of lasing initiation from below threshold due to an optical perturbation.

Finally, we examine the properties of the perturbing pulse itself as it propagates through the QDash amplifier. To this end, we have used a cross - frequency resolved optical gating (X-FROG) system [8,9] shown schematically in Fig. 3(a). The X-FROG scheme requires that the intense sampling pulse be fully characterized by a standard FROG measurement. The input pulse centered at $1585 \mathrm{~nm}$ had energy of $926 \mathrm{pJ}$ while the amplifier was biased below threshold at $192 \mathrm{~mA}$. Fig 3(b) shows the perturbing pulse which is a clear Gaussian like pulse with low chirp. Fig. 3 (c) shows the pulse after traversing the amplifier and initiated lasing. The original pulse is followed now by a second much stronger pulse which is delayed by approximately 200fs. Based on the knowledge of the instantaneous gain response detailed in [1], we understand the second peak to be an observation of self-induced amplification namely, amplification of the pulse trailing edge by TPA excited carriers originating from the leading edge. The observed effect can be interpreted as a direct measurement of the carrier-carrier scattering time. The delay of about $200 \mathrm{fs}$ is consistent with theoretical predictions [2].
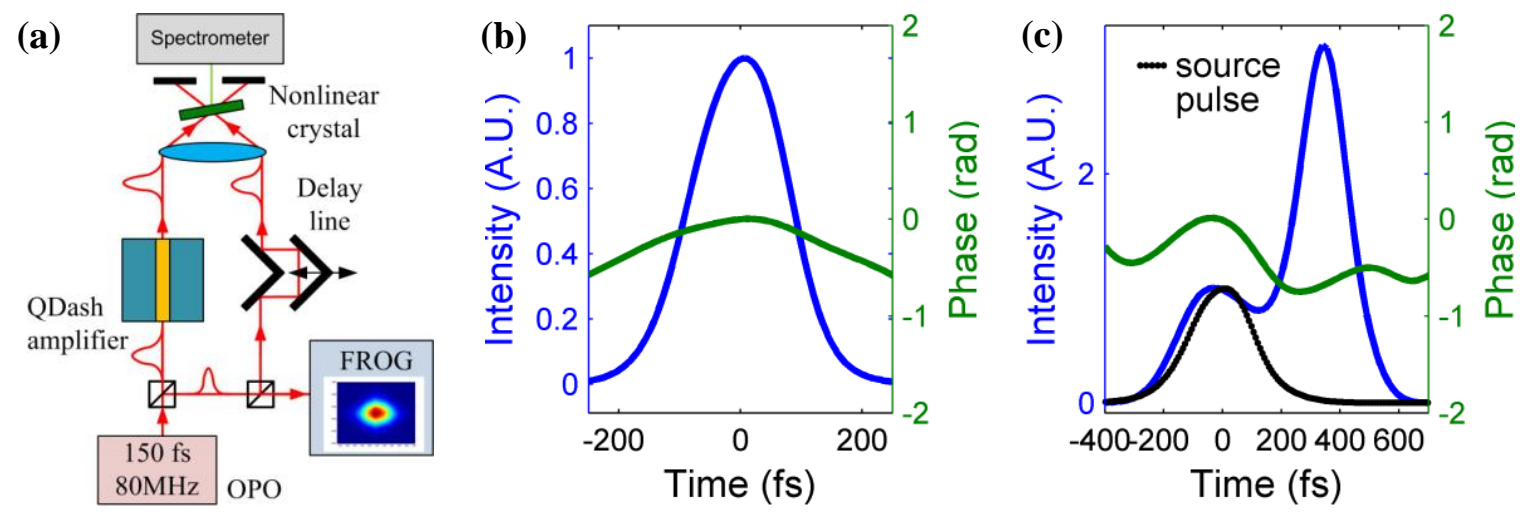

Fig. 3 - (a) The X-FROG scheme. (b) Retrieved source pulse. Intensity is normalized so that the peak reaches unity. (c) Retrieved pulse after propagating through the amplifier. The source pulse is marked for comparison. Intensity is normalized so that the first peak reaches value of unity.

To conclude, we have demonstrated a unique lasing mechanism in quantum dash gain media which relies on TPA, the wire like density of state function of the dashes and the gain inhomogeneity. Lasing at $1570 \mathrm{~nm}$ is initiated by short pulses centered at wavelengths which are either longer or shorter than $1570 \mathrm{~nm}$ with the laser threshold dropping as the pump wavelength increases. Detailed time domain characterization of the lasing output as well as XFROG measurements which characterize the perturbation pulse itself have been presented. The latter reveals a direct observation of the nearly instantaneous gain response and a direct measure of carrier-carrier scattering times.

This work was supported by the European commission through the project GOSPEL.

\section{References}

[1] A. Capua, G. Eisenstein, and J. P. Reithmaier, "A nearly instantaneous gain response in quantum dash based optical amplifiers," Appl. Phys. Lett. 97, 131108 (2010).

[2] H. Dery and G. Eisenstein, "Self-consistent rate equations of self-assembly quantum wire lasers," IEEE J. Quantum Electron. 40, 1398 (2004).

[3] A. Capua, G. Eisenstein, and J. P. Reithmaier, "Ultrafast cross saturation dynamics in inhomogeneously broadened InAs/InP quantum dash optical amplifiers," Appl. Phys. Lett. 98, 101108 (2011).

[4] A. V. Platonov, C. Lingk, J. Feldmann M. Arzberger, G. Bohm, M.-C. Amann, and G. Abstreiter, "Ultrafast switch-off of an electrically pumped quantum-dot laser," Appl. Phys. Lett. 81, 1177 (2002).

[5] D. H. Sutter, H. Schneider, S. Weisser, J. D. Ralston and E. C. Larkins, " Picosecond spectroscopy of optically modulated high-speed laser diodes," Appl. Phys. Lett. 67, 1809 (1995).

[6] E. Malić, K. J. Ahn, M. J. P. Bormann, P. Hövel, E. Schöll, A Knorr M. Kuntz and D. Bimberg, "Theory of relaxation oscillations in semiconductor quantum dot lasers," Appl. Phys. Lett. 89, 101107 (2006).

[7] M. Kauer, J. R. A. Cleaver, J. J. Baumberg and A. P. Heberle, "Femtosecond dynamics in semiconductor lasers: Dark pulse formation," Appl. Phys. Lett. 89, 1626 (1998).

[8] S. Linden, H. Giessen, and J. Kuhl, "XFROG - a new method for amplitude and phase characterization of weak ultrashort pulses," phys. stat. sol. (b) 206, 119 (1998).

[9] F. Romstad, P. Borri, W. Langbein, J. Mork, J.M. Hvam, "Measurement of pulse amplitude and phase distortion in a semiconductor optical amplifier: from pulse compression to breakup," Photon Tech Lett, 12, 1674 (2000). 